\title{
Oral multiunit pellet extended release dosage form: A review
}

\author{
*Vishal Sachdeva ${ }^{1}$, Md. Shoaib Alam², Ramesh Kumar ${ }^{2}$, Mahesh Kumar Kataria ${ }^{1}$ \\ ${ }^{1}$ Department of Pharmaceutics, Seth G.L. Bihani S.D college of Tech. Edu. Sriganganagar, Rajasthan, India \\ ${ }^{2}$ PDL - Formulation, Ranbaxy Laboratories Limited, Paonta Sahib, Sirmour - 173025, India
}

\begin{abstract}
Oral drug delivery is the most preferred route for the various drug molecules among all other routes of drug delivery, because ease of administration which lead to better patient compliance. So, oral extended release drug delivery system becomes a very promising approach for those drugs that are given orally but having the shorter half-life and high dosing frequency. Recent trends indicate that multiparticulate drug delivery systems are especially suitable for achieving extended release oral formulations with low risk of dose dumping, flexibility of blending to attain different release patterns as well as reproducible and short gastric residence time. The release of drug from pellets depends on a variety of factors including the carrier used to form pellets and the amount of drug contained in them. Consequently, pellets provide tremendous opportunities for designing new controlled and extended release oral formulations, thus extending the frontier of future pharmaceutical development.
\end{abstract}

Key Words: Extended release, pellets, dosage form.

\section{INTRODUCTION}

To the date, for every disease or disorder state of the patient, proper medication is of prime importance to maintain the patient in good health. To achieve this, the medicine or drug is administered conventionally by one or more of several well defined and popular routes of drug administration including oral, parenteral, rectal, alveolar, ocular and topical. Among these above mentioned popular routes, oral conventional route of drug administration lies at the top of the hierarchy of the conventional routes. It is a reasonable assumption that drug concentration at the site of action is related to drug plasma level and that, in the great majority of cases, the intensity of effect is some function of drug concentration at the target site. The objective of the most therapeutic regimens is to rapidly raise the plasma concentration to the required level and then to hold it constant for the desired duration of treatment. The extent to which this situation can be achieved depends on many factors, including the minimum effective concentration of the drug, the level at which side effects occur, the dose administered, the rate of drug release from the dosage form, the rate of elimination and the frequency of dosing. Provided that the dose size and frequency of administration are correct, therapeutic 'steady state' levels of the drug can be achieved rapidly and maintained by the repetitive administration of conventional oral dosage forms.

Traditionally patient only takes medication during the day time hours. Plasma levels can therefore fall to sub- therapeutic levels overnight. However, there are a number of major deficiencies of conventional dosage forms, few of which are listed here (Chien et al., 2002):

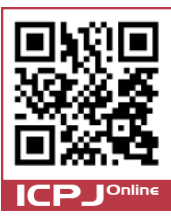

Drawbacks of conventional dosage forms

- Poor patient compliance, increased chances of missing the dose of a drug with short half-life for which frequent administration is necessary.

- The unavoidable fluctuations of drug concentration may lead to under medication or over medication.

- A typical peak-valley plasma concentration time profile is obtained which makes attainment of steadystate condition difficult.

- The fluctuations in drug levels may lead to precipitation of adverse effects especially of a drug with small Therapeutic Index (TI) whenever over medication occur.

\section{Extended release (ER) dosage form}

It is defined as those that allow at least a two folds reduction in frequent dosing compared to the drug presented in a conventional form (e.g., a solution or an immediate release dosage forms) (Chang and Robinson, 2002).

\section{Pellets}

Pelletization is an agglomeration process, that converts fine powder blend of drug(s) and excipients into small, free flowing, spherical units, referred to as pellets.

\section{Rationale of extended release pellets}

Pellets provide the development scientist with a high degree of flexibility during the design and development of oral dosage forms. They can be divided into desired dose strengths without formulation or process changes, and can also be blended to deliver incompatible bioactive agents simultaneously or particles with different release profiles at the same site or at different sites within the gastrointestinal tract (Patel et al., 2010).
Advantages of extended release pellets
- $\quad$ Reduce dosing frequency of drugs.
- Maintain therapeutic concentrations.
- $\quad$ Reduce the toxicity by slowing drug absorption. 
- The use of pellets avoids the high blood concentration.

- Extended release formulations have the potential to improve the patient compliance and convenience.

- Minimize the local and systemic side effects.

- Increase the stability by protecting the drug from hydrolysis or other degradative changes in gastrointestinal tract.

- Improvement in treatment efficacy.

- Minimize drug accumulation with chronic dosing.

- Improve the bioavailability of some drugs.

- Usage of less total drug.

- Improve the ability to provide special effects (Shargel and Andrew, 1999).

\section{DRUG PROPERTIES OF ER FORMULATIONS}

During design of extended release delivery systems, variables such as the route of drug delivery, the type of delivery system, the disease being treated, the patient, the length of therapy and the properties of the drug, are considered of particular interest to the scientist designing the system are the constraints imposed by the properties of the drug. These properties are classified as:

(a) Physicochemical

(b) Biological properties

These properties have the greatest effect on the behaviour of the drug in the delivery system and in the body. There is no clear cut distinction between these two categories since the biological properties of a drug are a function of its physicochemical properties. By definition, physicochemical properties are those that can be determined from in vitro experiments and biological properties will be those that result from typical Pharmacokinetic studies of the absorption, distribution, metabolism, and excretion (ADME) characteristics of a drug and those resulting from pharmacological studies (Robinson, 1970).

\section{Physicochemical Properties}
a) Dose Size
b) Aqueous Solubility and pKa
c) Partition Coefficient
d) Drug Stability
e) Molecular Size and Diffusivity
f) Drug Protein Binding

\section{Biological Properties \\ a) Absorption \\ b) Distribution \\ c) Metabolism \\ d) Elimination and Biological Half-Life \\ e) Margin of Safety}

\section{MULTIPARTICULATE AS ORAL ER DRUG DELIVERY SYSTEM}

These have gained much attention in the last two decades, due to their flexibility during formulation development, but also due to therapeutic benefits. In fact, multiparticulates present numerous advantages over single unit dosage forms as follows.

- Multiparticulates disperse in the gastro-intestinal tract,

- Maximizing absorption,

- Minimizing side effects,
- $\quad$ Reduces inter and intra-patient variability and avoid the risk of local irritation (Bechgaard and Nielsen, 1978).

Pellets are defined as geometrical agglomerates obtained from diverse starting materials (sucrose, starch, microcrystalline cellulose, etc) and can be produced by different process conditions (Ghebre, 1997). Pellets loaded with different drugs can be blended and formulated in a single dosage form. This allows the administration of two or more types of drugs that may or not be chemically compatible, at the same or different sites within the gastro-intestinal tract. Furthermore, pellets with different release rates from the same drug can be combined in a single unit dosage form in order to achieve the desired drug release profile (Karrout et al., 2009). Due to low surface area to volume ratio, ideal shape for film coating, good flowability, low friability, narrow particle size distribution, uniform and reproducible batches are obtained. Coated pellets can be compressed into tablets or filled into hard gelatin capsules as final dosage form. In order to achieve extended drug release, pellets can be directly coated or prepared with a polymer: drug solution or dispersion (matrix/matrix coated pellets) or loaded with drug and further coated with a polymeric solution or dispersion (reservoir/reservoir coated pellets) (Bodmeier, 1997).

\section{TYPES OF MULTIPARICULATE SYSTEM}

\section{A) Matrix Systems}

In matrix systems a polymer: drug solution or dispersion is granulated with excipients to form pellets or sprayed onto pellets in order to achieve extended drug release. The drug homogeneously distributed within the polymer is dissolved, dispersed or dissolved and dispersed. These systems present several advantages as follows

- $\quad$ Easy manufacture and low cost (1 step process),

- Lower risk of dose dumping (if the coating accidentally ruptures) and the

- Possibility of improvement of aqueous drug solubility.

Drug-polymer interactions can occur and bring benefits in terms of mechanical properties such plasticizing effect. The main disadvantages include fast initial release and incomplete release in a defined time (Huang and Brazel, 2001). The latter could be avoided by coating sugar cores with different polymer: drug ratios, in which the drug was more concentrated in deeper layers of the matrix and so counteracting for the increased diffusion pathway. In addition, matrix systems were found suitable to control drug release of a highly soluble drug (Scott and Hollenbeck, 1991).

Matrix solutions, matrix dispersions and drug release mechanisms

In matrix systems, the drug and polymer are dissolved or dispersed in a common solvent and upon solvent evaporation, a solid solution (drug dissolved in the polymer) or a solid dispersion (drug dispersed in the polymer) or a combination of both is obtained. If the initial drug concentration is below drug solubility in the polymer, drug is dissolved and drug release is mainly extended by drug diffusivity in the polymer.

\section{B) Reservoir Coated Systems}

A reservoir coated system consists of a drug layered core surrounded by a polymer. The major advantages of this system rely in the fact that very high drug loadings can be 

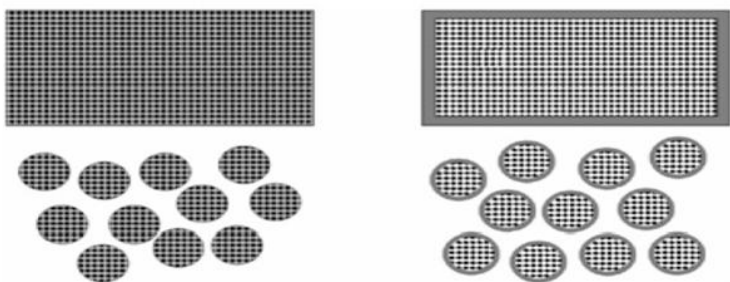

Figure 1: Schematic presentation of: (A) matrix system and (B) reservoir system (Black: Drug, Gray: Release controlling polymer, white: excipients).
(A)

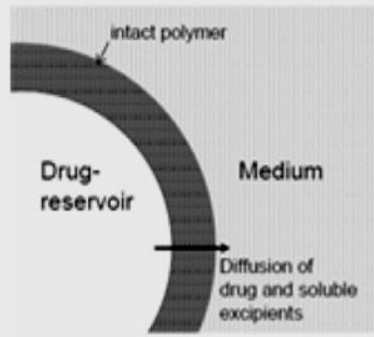

(C)

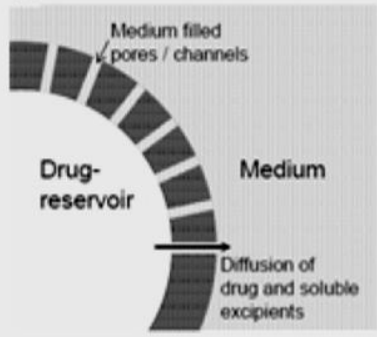

(B)

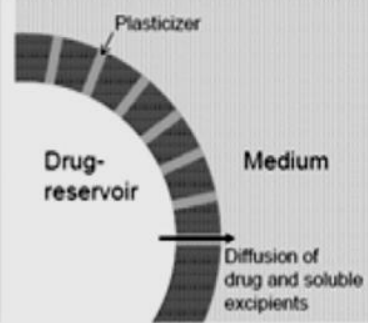

(D)

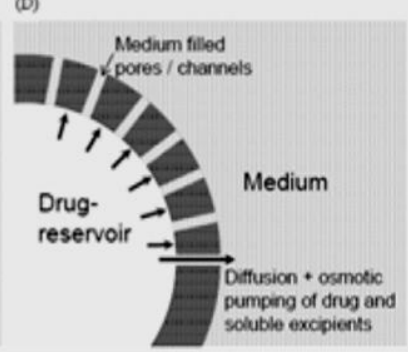

Figure 3: Schematic presentation of typical release mechanism of coated pellets.

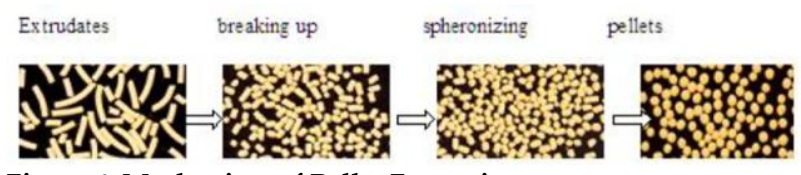

Figure 6: Mechanism of Pellet Formation.

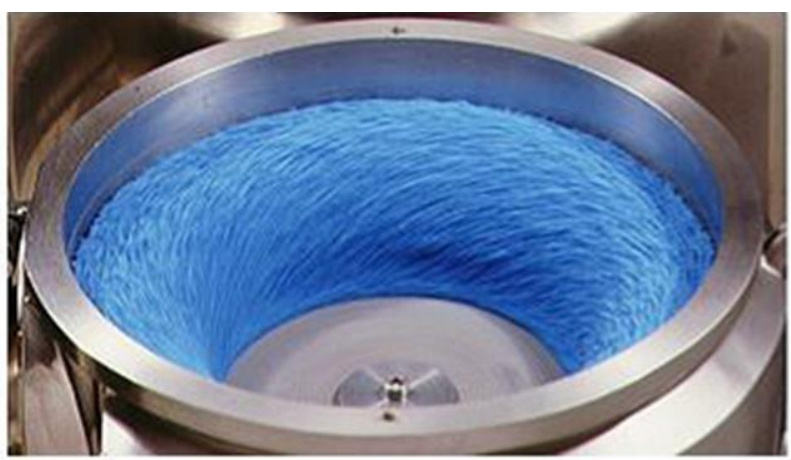

Figure 7: Spheronization Process.
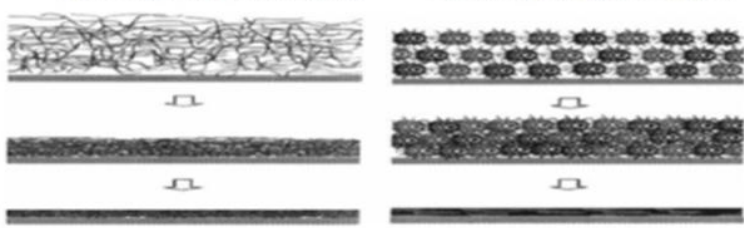

Figure 2: Schematic presentation of (A) the film forming mechanism from organic Polymer solution (B) the film forming mechanism from aqueous polymer dispersion.

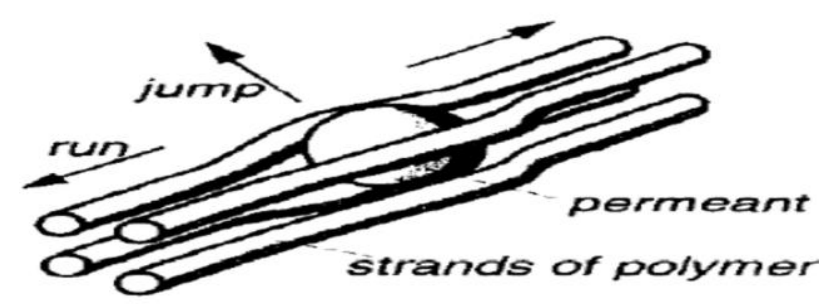

Figure 4: The jump-and-run model of per meant diffusion through intact polymer.

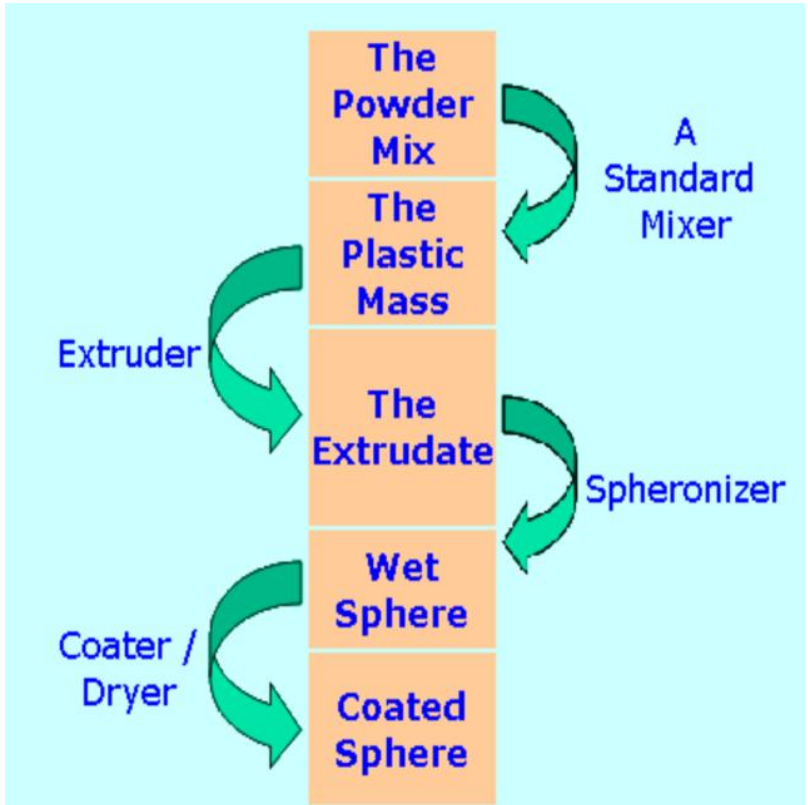

Figure 5: Overview of the Formulation of ER Pellets.

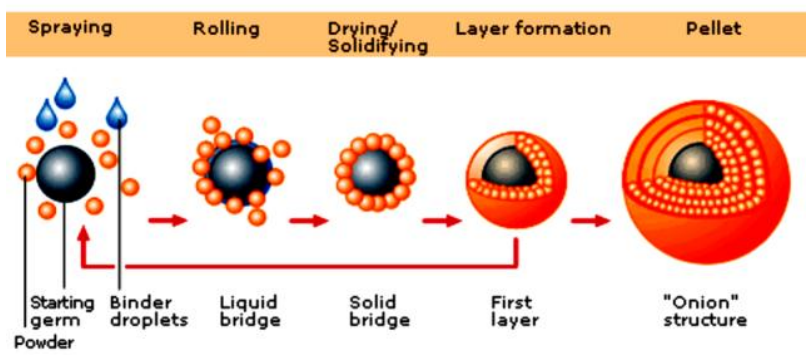

Figure 8: Principle of powder layering process. 
used and variable drug release profiles can be obtained, by just varying the type of polymeric membrane.

\section{Aqueous coating and organic coating}

Pellets can be coated with an aqueous polymeric dispersion or an organic solution in order to achieve extended drug release. Organic coatings present many disadvantages as the dependence of viscosity on molecular weight and the concentration of polymer used. In contrast, aqueous polymer dispersions are characterized by low viscosity even at high solid contents, leading to a decrease in coating process time. (McGinity, 1997) Organic solutions present additional disadvantages like the presence of residual solvents in the coating that can create changes in film properties, environmental pollution and explosion hazards. As a result, the use of aqueous polymeric dispersions is preferred for pharmaceutical coatings. However, film formation mechanisms (aqueous versus organic) are very different. With organic polymer solutions, polymer macromolecules are dissolved and this can create a high viscosity solution. During solvent evaporation, an intermediate gel-like phase is formed. After complete solvent evaporation, a polymeric film is obtained (Figure 2: A).

In contrast, film formation from aqueous dispersions is a more complex process (Scott and Hollenbeck, 1991). During drying of aqueous dispersions, polymer particles come into contact with each other in a closed packed order. The high interfacial surface tension between air and water leads to the formation of a layer of polymer spheres filled with water. The particle fusion or coalescence is then possible when the capillarity forces (air water interfacial tension) are strong enough. Usually the coating process is performed at sufficient high temperatures to guarantee softness of the discrete polymer particles. The softening is related to the glass transition temperature (Tg) of the polymer. A curing step (post coating thermal treatment) is carried out after coating process to assure complete film formation and avoid further gradual coalescence. The aqueous dispersions can have additional ingredients as surfactants that act as stabilizers during the production process. Other compounds as plasticizers and anti-taking agents are used to enhance the coating process and film properties. Plasticizers are added to promote the polymer particle coalescence, softening the particles and reducing minimum film formation temperature (MFT). Film formation is related to glass transition temperature of the polymer or minimum film formation of the aqueous dispersion. The MFT is the minimum temperature above a continuous film is formed during drying under standardized conditions. Below this temperature the dry latex is opaque and powdery; however these conditions are different from drying during coating.

Actually, water can decrease Tg of the some polymers (due to its plasticizing effect) and in this case the MFT is lower than the Tg of the polymer. Tg and MFT shows a linear relationship between different polymer/plasticizer concentrations (Lippold and Monells, 2001).

\section{Drug release mechanisms}

The mechanism controlling drug release from reservoir coated pellets is often a complex process and it depends on coating type and thickness, drug type and core type. One of the mechanisms is diffusion through the continuous polymer film surrounding the drug loaded core (Munday and Fassihi 1989). Firstly, water penetrates through the coating until reaches the pellet core. After- wards, drug is dissolved and released. The drug is released due to the concentration gradient inside the pellet $(\mathrm{Ci})$ versus outside the pellet. In the case of perfect sink conditions the amount of drug released (dM) within a certain time period $(\mathrm{dt})$ can be calculated as follows (according to Fick's law of diffusion):

$$
\frac{d M}{d t}=D m \cdot A \cdot K \cdot \frac{C i}{d}
$$

Dm is the apparent diffusion coefficient of the drug in the polymeric film, A the surface available for diffusion, $\mathrm{K}$ the partition coefficient of the drug (aqueous phase polymeric phase), and d denotes the thickness of the film coating (Tang et al., 2000). Unfortunately, Fick's Law (which was only ever intended to describe diffusion in binary mixtures) cannot be extended to drug release from reservoir pellets that easily. The diffusivity for example is assumed to be constant in homogeneous, intact polymer films. However, in reality many polymers swell upon contact with medium which is known to gradually increase the diffusivity over time. In addition most polymers contain crystalline regions in which drug diffusion is negligible.

Drug diffusion in the amorphous regions of polymers has been described by the so-called 'jump-and-run'model. It was proposed that the amorphous segments in polymers contain homogeneous, semi-crystalline structures of polymer molecules which are aligned in parallel. Permeants like the diffusing drug 'run' along the tube between parallel polymer chains until reaching a 'dead-end' (a crystalline region or a point of high chain entanglement). There they are forced to 'jump' from one tube to the next, pushing and bending the polymer chains apart (Figure 4). Drug release can occur through water filled pores (Figure 3: B, C). These pores can be due to leaching of water soluble compounds into the release medium or due to cracks formed by high hydrostatic pressure generated inside these systems upon water uptake. Drug release can be described as follows:

$$
\frac{d M}{d t}=D p \cdot A \cdot \frac{\varepsilon}{\tau} \cdot \frac{C i}{d}
$$

Where Dp is the diffusion coefficient of the drug in the aqueous phase present in the channels and pores, $\varepsilon$ the volume fraction of the pores, $\tau$ the tortuosity of the channels (Ozturk et al., 1990). Another possible mechanism controlling drug release from coated pellets is due to osmotic effects (Figure 3: D). For this mechanism to occur an osmotic active core should be surrounded by semi permeable membrane and a difference in osmotic pressure between the inner and outer side of the membrane.

Osmotically driven release depends on the porosity of the polymeric membrane and the osmotic pressure of the sugar core and the drug. Upon water uptake, drug is pushed out via pores in the coating. Drug release can be described as follows (Ozturk et al., 1990):

$$
\frac{d V}{d t}=\frac{A \theta \Delta \pi}{I}
$$

Where $\mathrm{dV} / \mathrm{dt}$ denotes the water flow, A the membrane surface area, 1 the membrane thickness, $\theta$ the permeability of the polymeric membrane, and $\Delta \pi$ the difference in 
osmotic pressure (neglecting the counteracting hydrostatic pressure). The overall drug release rate from coated pellets may be governed by one of the above mechanism or a combination of them. Parameters as core and coating swelling also contributes to the drug release rate (FrohoffHulsmann et al., 1999). The type of drug can strongly affect the resulting drug release rates. Ibuprofen diffused through the coating (due to high solubility in the polymer) while chlorpheniramine maleate diffused through micro-channels in Aquacoat coated pellets, resulting from osmotic pressure developed by the core. Drug release rate can be affected by changes in surface area (during dissolution study) of the pellets. The coating level also changes the mechanism of drug release. At low coating levels, drug release occurred through pores in the coating, while at high coating levels drug release rate was extended by diffusion through the coating. Consequently the mechanism controlling drug release at higher coating levels was not just dependent on drug solubility but also on the polymer/dissolution medium partitioning coefficient of the drug (Sadeghi et al., 2000).

Drug release mechanism from ethyl cellulose coatings with pore formers was investigated by several researchers. At lower pore former (HPMC) contents, drug release occurred through osmotic pumping, but above a certain value diffusion also contributed to overall drug release. Addition of small amounts of polyvinyl alcohol polyethylene glycol graft copolymer to ethylcellulose coatings was found to control drug release from coated pellets irrespective of the drug solubility and type of core formulation. The mechanism controlling drug release was shown to be diffusion through intact polymeric membranes.

The glass transition temperature of the polymer also affects the drug release mechanism. With water soluble plasticizers, the polymer was in glassy state after plasticizer migration and drug diffused through water filled pores. With water insoluble plasticizers, the polymer was in the rubbery state and a two phase release mechanism was found. In the first phase drug was released through pores created by leaching of HPMC and in the second phase pore shrinking occurred leading to a decrease of free volume in the polymer chains (Bodmeier and Paeratakul, 1993).

The type of coating technique (organic versus aqueous) was found to contribute to drug release mechanism in different ways. Drug release mechanism from coating with blends of a water-insoluble (ethylcellulose) and an enteric polymer (ethylcellulose: methacrylic acid ethylacrylate copolymer, Eudragit L) occurred by diffusion through the intact polymeric films and/or waterfilled cracks. However, lower hydrostatic pressures were necessary to induce crack formation within aqueous coatings. Organic coatings were mechanically strong with high degree of polymer-polymer interpenetration and thus higher hydrostatic pressure was required to induce crack formation.

The polymer particle size affects the film coating structure and properties. Blends of aqueous dispersions of a water-insoluble and an enteric polymer, ethylcellulose and Hydroxypropyl methyl cellulose acetate succinate (HPMCAS) and Eudragit L were used as coating materials to control theophylline release from matrix pellets. Drug releases were similar for both types of blends in $0.1 \mathrm{M}$ $\mathrm{HCl}$, but significant differences were observed in phosphate buffer $\mathrm{pH}$ 7.4. Eudragit L particles are smaller than HPMC particles (nano- vs. micrometer size range) and more effectively hinder the formation of a continuous
Table 1: properties of drugs to be considered for extended release (Lee and Robinson, 1978; Lee and Good, 1987).

\begin{tabular}{|c|c|}
\hline Suitable Drugs & Non-suitable Drugs \\
\hline $\begin{array}{l}\text { Physicochemical properties: } \\
\text { 1.Compounds with low MW }\end{array}$ & \multirow{2}{*}{$\begin{array}{l}\text { 1. Large molecular } \\
\text { size/weight (proteins and } \\
\text { peptides for oral) }\end{array}$} \\
\hline $\begin{array}{l}\text { 2.Good aqueous solubility, } \mathrm{pH} \\
\text { independent (Penotoxyphylline) }\end{array}$ & \\
\hline $\begin{array}{l}\text { 3.With non-aqueous solubility (for } \\
\text { Parenteral; Steroids) }\end{array}$ & $\begin{array}{l}\text { 2. Very low aqueous } \\
\text { solubility }(0.1 \mathrm{mg} / \mathrm{ml})\end{array}$ \\
\hline $\begin{array}{l}\text { 4.Unionized (at least } 0.1-5 \% \text { ) in GI } \\
\text { tract }\end{array}$ & $\begin{array}{l}\text { 3. Largely in ionized form } \\
\text { in the G.I. tract. }\end{array}$ \\
\hline $\begin{array}{l}\text { 5.Very weak bases } \mathrm{pKa}<5.0 \\
\text { (Theophylline } \mathrm{pKa}=0.7 \text {, Diazepam } \\
\text { pKa = 3.7) }\end{array}$ & $\begin{array}{l}\text { in the G.1. tract. } \\
\text { 4. Strong bases (pKa> } \\
\text { 11.0) e.g.: Guanethidine }\end{array}$ \\
\hline $\begin{array}{l}\text { 6.Very weak acids } \mathrm{pKa}>8.0 \\
\text { (Pentobarbital } \mathrm{pKa}=8.1 \text { ) Unionized }\end{array}$ & $\begin{array}{l}\text { 5. Strong acids }(\mathrm{pKa}<2.5) \\
\text { eg: Cromolyn sodium }\end{array}$ \\
\hline
\end{tabular}

at all $\mathrm{pH}$, absorb well

7. Moderately weak acids pKa 2.5-7.5 Aspirin (3.5), Ibuprofen (4.4).

8. Moderately weak bases (pKa 5.0-

11.0), Codeine (8.2) Ionization

depends on $\mathrm{pH}$

Pharmacokinetic properties

(Chien, 1992):

1. Short half-life (2-5 hr) e.g., theophylline (4hr) Sodium diclofenac $(2 \mathrm{hr})$ Nifedipine $(2.5 \mathrm{hr})$, Diltiazem(3.5 hr), Glipizide (3.4 hr)

2. Well absorbed from all regions of GI tract

1. Slow absorption

2. Carrier mediated transport (several B vitamins)

3. Site specific absorption (Vit B12)

4. Degradation in g.i. tract (Nitroglycerine, Penicillin G, Erythromycin)

5. First pass hepatic metabolism (Nitroglycerin, Propranolol)

6. That induce or inhibit metabolism (e.g., Rifampicin, Barbiturates, Allopurinol, PAS)

Pharmacodynamic property:

1. Therapeutic range of blood

1. Having large dose conc.-Wide enough

and mechanically stable ethylcellulose network. Ethyl cellulose structures remaining upon HPMC leaching are mechanically stronger and drug release is extended by diffusion through the polymeric remnants. In contrast, ethylcellulose structures remaining after enteric polymer leaching at high $\mathrm{pH}$ are mechanically much weaker in the case of Eudragit L. Upon exposure to phosphate buffer, water-filled cracks are formed, through which the drug rapidly diffuses out (Siepmann et al., 2005).

\section{Marketed products of MUPS}

Losec MUPS (Multiple Unit Pellet Systems), consisting of microencapsulated drug granules tableted with excipients is the second highest selling pharmaceutical drug product in Sweden in the year 2002. Different marketed products are tabulated in table 2 (Sirisha et al., 2012).

\section{Curing}

After coating process and even with a product temperature $10-20^{\circ} \mathrm{C}$ above the MFT, complete film formation may 
Table 2: Different marketed products of MUPS.

\begin{tabular}{|c|c|c|c|}
\hline Product & Company & Drug & Type \\
\hline Losec MUPS & $\begin{array}{l}\text { Astra } \\
\text { Zeneca }\end{array}$ & $\begin{array}{l}\text { Omeprazole } \\
\text { magnesium }\end{array}$ & $\begin{array}{l}\text { Delayed } \\
\text { Release }\end{array}$ \\
\hline Esomeprazole & $\begin{array}{l}\text { Astra } \\
\text { Zeneca }\end{array}$ & $\begin{array}{c}\text { Esomeprazole } \\
\text { Magnesium }\end{array}$ & $\begin{array}{l}\text { Delayed } \\
\text { Release }\end{array}$ \\
\hline Toprol XL & $\begin{array}{l}\text { Astra } \\
\text { Zeneca }\end{array}$ & Metoprolol tartrate & $\begin{array}{l}\text { Extended } \\
\text { Release }\end{array}$ \\
\hline PrevacidSoluTab & Takeda & Lansoprazole & $\begin{array}{l}\text { Delayed } \\
\text { Release }\end{array}$ \\
\hline Theodur & Key & Theophylline & $\begin{array}{c}\text { Extended } \\
\text { Release }\end{array}$ \\
\hline
\end{tabular}

not be achieved. Thus a short thermal treatment is required to complete polymer particle coalescence. At curing temperatures above the glass transition temperature, the mobility of the polymer chains increases and latex coalescence is accelerated. The curing step may be performed in an oven or in the fluidized bed coater immediately after the coating process. Too low curing temperatures can lead to incomplete film formation, whereas too high temperatures can lead to excessive tackiness and agglomeration of the solid dosage forms. The curing step can be performed at several temperatures or different times and in the presence of extended humidity. All these factors can potentially affect drug release rate. The slower release rates with increasing curing time were attributed to greater polymer particles coalescence (Bhattacharjya and Wurster, 2008). In another study, the curing temperature and time were investigated. Drug release decreased with increasing temperature. At $30^{\circ} \mathrm{C}$, the decrease in drug release was small and not affected by the curing time. When temperature and time of curing were increased, the resulting changes in drug release rate increased. It was suggested that at higher temperatures, more polymer molecules can overcome the energy barrier and reach a stable state, reflected by the slower release. On the contrary, at low curing temperatures, few molecules can achieve a stable state, meaning that changes in drug release are expected to occur slowly over time until the stable state is reached (Lin et al., 2003). Extended humidity can be used during the curing step. The presence of humidity was more effective to complete film formation than without. Water facilitates polymer particle coalescence and it acts as plasticizer for many polymers. High content of plasticizer can minimize the curing effect, however there is a limit of plasticizer concentration to avoid problems as stickiness during coating process or forming agglomerates of pellets during curing. The curing effect on drug release can change depending on the type plasticizer and coating level. For example, drug release decreased with increasing harshness (time, temperature and relative humidity) of curing conditions, when using triethyl citrate \& acetate as plasticizer (Yang et al., 2010).

\section{Storage stability}

Although the curing step is performed in order to complete film formation, drug release rate was reported to decrease especially under elevated humidity. This was mainly attributed to further gradual polymer coalescence, leading to denser films and decreased permeability for water and drug (Wu et al., 2000). Changes in drug release profiles were also observed with high glass transition temperature polymers. Faster drug release may be caused by brittle films or the formation of micro-ruptures in the film coat during storage. Thermal humidity curing was found to help to enhance coalesce of polymeric films, however presence of high levels of humidity during storage can destabilize films, originating changes in drug release rate over time (Liu and Williams, 2002).

\section{FORMULATION METHODS}

\section{Extrusion Spheronization Process}

The concept of multiparticulate dosage forms introduced in the 1950's with the increasing use of multiparticulate extended release (CR) oral dosage forms, in recent times there has been a rise in interest in the methods of preparing these dosage forms. A method that has gained increased usage over the past few years is that of extrusion and spheronization.it has extensively as a potential technique and also as a future method of choice for preparation of multiparticulate CR dosage forms. This is a multi-step process involving dry mixing, wet granulation, extrusion, spheronization, drying and screening. The first step is dry mixing of the drug and excipients in a suitable mixer followed by wet granulation, in which the powder is converted into a plastic mass that is easily extruded. The extruded strands transferred into a spheronizer, where they are instantaneously broken into short spherical rods on contact with the rotating friction plate and pushed outward and up the stationary wall of the processing chamber by centrifugal force. Finally, owing to gravity, the particles fall back to friction plate, and the cycles repeated until the desired sphericity achieved. Extrusion-spheronization is a multistep process involving a number of unit operations and equipment. However, the most critical part of processing equipment dictates the outcome of overall quality of pellets (Kumar et al., 2012).

\section{Extrusion}

Shaping of the wet mass into long rods is called as extrusion. A variety of extruders, which differ in design features and working principles, are currently on market and can be classified as screw-fed extruder, gravity-fed extruder and ram extruder. Screw-fed extruder have screws that rotate along the horizontal axis and hence transport the materials horizontally, they may axial or radial screw extruders. The product temperature extended during extrusion by jacketed barrels. In radial extruders, the transport zone is short, and the material extruded radially through screens mounted around the horizontal axis of the screws. Gravity-fed extruders include the rotating cylinder and rotating gear extruders, which differ primarily in the design of two counterrotating cylinders. In the rotating cylinder extruder, one of the two counter rotating cylinders is hallow and perforated, whereas the other cylinder is solid and acts as a pressure roller. In ram extruders, piston displaces and forces the materials through a die at the end. Ram extruders preferred during formulation development they designed to allow for measurement of the rheological properties of formulation. In an extrusion-spheronization process, formulation components such as filler, lubricants and $\mathrm{pH}$ modifiers play a critical role in producing pellets with desired attributes. The granulated mass must plastic and sufficiently cohesive and self-lubricating during extrusion. During the spheronization step, it is essential that the extrudates break at appropriate length and have sufficient surface moisture to enhance the formulation of uniform spherical pellets. Excipients play an important role during extrusion spheronization than during with other pelletization process. They facilitate extrusion and determine the spherecity of the wet pellets, impart 
strength and integrity of the pellets. Microcrystalline cellulose (MCC) is the most commonly used excipient in extrusion spheronization it leads to the formation of round spheres with desirable characteristics (Fridrun et al., 2009).

During spheronization, moisture entrapped in the MCC microfibrils adds plasticity to the extrudates into spherical pellets. The pellet properties can be affected by many operational variables during the extrusion stage, the spheronization stage, or the drying stage. Both drying technique and drying temperature have a considerable effect on the pellet structure and properties. The variables that affect the final pellet qualities are screen pressure, screen hole diameter, extruder type and speed, the type of friction plate, and spheronization time, speed and load. There is considerable interaction between spheronization time and spheronization load. With small and large spheronization loads, the yield of large pellets increases with longer spheronization time, an effect that is exacerbated by faster spheronization speed. Unsuitable processing parameters lead to pellet with poor qualities (Lieven et al., 2009).

\section{Spheronization}

During the third phase of extrusion spheronization process the extrudates dumped on to the spinning plate of the spheroniser, call the friction plate, where the extrudate broken up into smaller cylinders with a length equal to their diameter, those plastic cylinders rounded due to frictional forces. In the spheronization process different stages are distinguished depending on the shape of the particles, i.e.; starting from a cylinder over a cylinder with rounded edges, dumbbells and elliptical particles to eventually perfect spheres. Baert and remon (1993) suggested that another pellet forming mechanism might exist. In this mechanism twisting of a cylinder occurs after the formation of cylinders with rounded edges, finally resulting in the breaking of the cylinder into two distinct parts. Both parts have round and flat side. Due to rotational and frictional forces involved in the spheronization process the edges of the flat side fold together like a flower forming the cavity observed in certain pellets. The spheronization of a product usually takes 2-10 minutes. A rotational speed of friction plate in the range between 200 and 400 RPM would be satisfactory to get highly spherical pellet. This statement is in a sharp contrast with most reports indicating the use of spheronization speeds exceeding 400 RPM. This contradiction is explained by the fact that not the absolute speed is important but the speed in combination with the diameter of the friction plate. From those two parameters the plate peripheral velocity is calculated and this data should be compared instead of absolute rotational speed of the friction plate. The friction plate has a grooved surface to increase the frictional forces. Two types of geometry of the grooves exist, cross hatch geometry where the grooves from right angles and radial geometry where a radial pattern used (Newton et al., 2002).

\section{Layering process}

Layering processes involve loading solid inert cores with drugs and/or excipients. Inert cores, placed in a suitable vessel such as a coating pan or a fluid bed, may be layered according to different methods. Some methods consist of spraying onto the cores a solution/suspension containing both drug and binding agent. Others are based on layering the drug directly in powdery form where drug loading occurs by gravity and adhesion is ensured by a liquid binder sprayed onto the cores.

The layering process is particularly suitable for production of small drug loaded units, multiples of which are placed into capsules for patient delivery. In the case of spherical inert cores such as non-pareils, the layering techniques from solution/suspensions produce homogeneous drug loaded particles, which retain an approximately spherical shape. They are therefore particularly suitable for successively film coating to build up the particle with the aim of providing a desired drug release profile (Cornelli et al., 2007).

Powder layering involves the deposition of successive layers of dry powder of drug or excipients or both on performed nuclei or cores with the help of a binding liquid. Because powder layering involves the simultaneous application of the liquid and dry powder, it generally requires specialized equipment. Pieces of equipment revolutionized powder layering processing as a pelletizing techniques are- tangential spray or centrifugal fluid bed granulators. In case of tangential spray the rotating disk and fluidization air provides proper mixing. With a double wall centrifugal granulator, the process is carried out in the open and closed position. With powder layering, the inner wall is closed so that simultaneous application of liquid and powder could proceed until the pellets have reached the desired size. The inner wall is then raised, and the spheres enter the drying zone. The pellets are lifted by the fluidization air up and over the inner wall back in to forming zone. The cycle is repeated until the desired residual moisture level in the pellets is achieved. The principle of powder layering process with different steps is completely illustrated in figure 8 (Patel et al., 2010).

\section{CONCLUSION}

In this Review we Concluded that Pellets are for pharmaceutical purposes and are produced primarily for the purpose of oral extended-release dosage forms having gastro resistant or extended-release of extended release properties or the capability of site-specific drug delivery. For such purposes, coated pellets are administered in the form of hard gelatin capsules or in tablets. As drugdelivery systems extended release pellets become more sophisticated, the role of pellets in the design and development of dosage forms is increasing. Formulation of drugs in multiple-unit dosage forms, such as extended release coated pellets filled in capsules or compressed into tablets, offers flexibility as to target-release properties. The safety and efficacy of the formulation is higher than that of other dosage forms

\section{REFERENCES}

Bechgaard H and Nielsen G, (1978).Extended release multiple-units and single-unit doses a literature review. Drug Development and Industrial Pharmacy 4 (3) 53 - 67. [DOI]

Bhattacharjya S, Wurster D (2008). Investigation of the Drug Release and Surface Morphological Properties of Film- Coated Pellets, and Physical, Thermal and Mechanical Properties of Free Films as a Function of Various Curing Conditions, AAPS Pharmaceutical Science and Technology 9 449-457. [DOI] PMid:18431665 PMCid: PMC2976940

Bodmeier R, Paeratakul O (1993). Dry and wet strengths of polymeric films prepared from an aqueous colloidal polymer dispersion, Eudragit RS30D, International Journal of Pharmaceutics 96 (1) 129-138. [DOI]

Bodmeier R. (1997). Tableting of coated pellets, review, European Journal of Pharmaceutics and Biopharmaceutics 43 (5) 1-8 [DOI]

Chang RK, Robinson JR. (1990) Tablets In: Lieberman, HA, Lachman L. Pharmaceutical DosageForms: vol. 3 New York, Marcel Dekker.

Chien Y W, (1992). Novel drug delivery system., 2nd edition, New york. Marcel Dekker Inc. 
Chien YW, Lin S, Swarbrick J \&Boylan J (2002). Drug Delivery- Extended Release In "Encyclopediaof Pharmaceutical technology: vol. INew York, Marcel Dekker

Cornelli, (2007). Layering process for multiparticulate dosage form, United States Patent 6569462

Fridrun P. (2009). The influence of non-ionic surfactants on the rheological properties of drug/microcrystalline cellulose/water mixtures and their use in the preparation and drug release performance of pellets prepared by extrusion/spheronization, European Journal of Pharmaceutical Sciences37 (8) 334-40.

Frohoff-Hulsmann MA (1999). Aqueous ethyl cellulose dispersion containing plasticizers of different water solubility and hydroxypropyl methyl-cellulose as coating material for diffusion pellets II, properties of sprayed films, European Journal of Pharmaceutics and Bio pharmaceutics 48(9) 67-75. [DOI]

Ghebre S. (1997).Multiparticulate Oral Drug Delivery, first edition New York Marcel Dekker Inc. [Link]

Huang X, Brazel C (2001). On the importance and mechanisms of burst release in matrix extended drug delivery systems, Journal of Control Release73(2) 128- 136

James McGinity(1997). Aqueous Polymeric Coatings for Pharmaceutical Dosage Forms, second edition New York Marcel Dekker Inc.

Karrout Y. et al (2009). Novel polymeric film coatings for colon targeting Drug release from coated pellets, European Journal of Pharmaceutical Science 37 (1) 427-433 [DOI] PMid:19491034

Kumar S.(2012). Formulation and Evaluation of Multiunit Pellet System of Venlafaxine Hydrochloride, Journal Of Pharmaceutical And Biomedical Sciences $182-3$

Lee P, Good W. (1987). Overview of Controlled Release Drug DeliveryIn Controlled Release Technology: Pharmaceutical Applications. American ChemicalSociety, Washington D.C. [DOI]

Lee VHL, Robinson JR (1978). Sustained and Controlled Release Drug Delivery Systems vol. 6 New York, Marcel Dekker Inc.

Lieven B. (2009). Improved bioavailability of darunavir by use of $\kappa-$ carrageenan versus microcrystalline cellulose as pelletisation aid European Journal of Pharmaceutics and Bio pharmaceutics, 72614-20.

Lin AY. (2003). A Study of the Effects of Curing and Storage Conditions on Extended Release Diphenhydramine $\mathrm{HCl}$ Pellets Coated with Eudragit@NE30D. Pharmaceutical Development and Technology 8277 287. [DOI] PMid:12901693

Lippold BC and Monells PR 2001, Control and stability of drug release from diffusion pellets coated with the aqueous quaternary Polymethacrylate dispersion Eudragit RS 30 D. Pharmazie vol. 56 pp 477-483 PMid:11446169
Liu J, Williams R (2002). Properties of heat-humidity cured cellulose acetate phthalate free films, European Journal of Pharmaceutical Science 17 31-41. [DOI]

Munday DL, Fassihi AR (1989).Extended release delivery: Effect of coating composition on release characteristics of mini-tablets. International Journal of Pharmaceutics52 (1) 109-114. [DOI]

Newton J.M. (2002). Factors influencing the physical characteristics of pellets obtained by extrusion and spheronization, International Journal of Pharmaceutics,232 91-106. [DOI]

Ozturk AG et al (1990), Mechanism of release from pellets coated with an ethylcellulose-based film, Journal of Control Release vol. 14 203-213. [DOI]

Patel P. (2010). Pellets: A General Overview. International Journal of Pharma World Research 1 (2) 1-15

Ritger P, Peppas N (1987). A simple equation for description of solute release, Fickian and non-Fickian release from non-swell able devices in the form of slabs, spheres, cylinders or discs, Journal of Control Release5(1) 23-36

Robinson M. (1970). Sustained Action Dosage Forms TheTheory and Practice of Industrial Pharmacy 2nd edition Philadelphia, Lea and Febiger.

Sadeghi F (2000). Comparative Study of Drug Release from Pellets Coated with HPMC or Surelease, Drug Development and Industrial Pharmacy 26 651-660. [DOI] PMid:10826113

Scott DC, Hollenbeck RG (1991).Design and manufacture of a zero-order extended release pellet dosage form through non uniform drug distribution in a diffusional matrix, Pharmaceutical Research 8(5) 156161 [DOI] PMid:2023862

Shargel L, Andrew BC. (1999). Applied Biopharmaceutics and Pharmacokinetics. New York $4^{\text {th }}$ Edition Prentice-Hall International.

Siepmann F. (2005). Blends of aqueous polymer dispersions used for pellet coating, Importance of the particle size, Journal of Control Release105226- 239

Tang L et al (2000). Drug release from film-coated chlorpheniramine maleate nonpareil beads, effect of water-soluble polymer, coating level, and soluble core material. Pharmaceutical Development and Technology5383-390

Wu C. (2000). Influence of relative humidity on the mechanical and drug release properties of theophylline pellets coated with an acrylic polymer containing methylparaben as a non-traditional plasticizer, European Journal of Pharmaceutics and Bio pharmaceutics 50 277-284 [DOI]

Yang QW. (2010).curing of aqueous polymeric film coatings: Importance of the coating level and type of plasticizer, European Journal of Pharmaceutics and Bio pharmaceutics 74(3) 362-370. [DOI] PMid:19895886 